\title{
Interleukin-6 Induces Epithelial-Mesenchymal Transition in Breast Cancer Cells
}

\author{
Juan Francisco Alvarado-Muñoz ${ }^{1, \#, ~ J o s e ́ ~ E s p a r z a-L o ́ p e z ~ 2,4, \#, ~ E l i z a b e t h ~ E s c o b a r-A r r i a g a ~}{ }^{3}$, \\ Eliseo Neftalí De La Cruz-Escobar ${ }^{1}$, Eucario León-Rodríguez ${ }^{1}$ and María de Jesús \\ Ibarra-Sánchez ${ }^{2,4, *}$
}

\begin{abstract}
1Departamento de Hematología y Oncología, Instituto Nacional de Ciencias Médicas y Nutrición Salvador Zubiran; ${ }^{2}$ Unidad de Bioquímica, Instituto Nacional de Ciencias Médicas y Nutrición Salvador Zubirán; ${ }^{3}$ Centro Oncológico, Hospital Ángeles del Pedregal; ${ }^{4}$ Red de Apoyo a la Investigación, Coordinación de la Investigación Científica, Universidad Nacional Autónoma de México-Instituto Nacional de Ciencias Médicas y Nutrición Salvador Zubirán
\end{abstract}

Received for publication: 25 October 2016; accepted for publication: 25 February 2017

Available online: 4 August 2017

\section{KEYWORDS \\ Breast cancer; \\ Interleukin 6; \\ Epithelial- \\ Mesenchymal \\ Transition}

\begin{abstract}
Background: Breast cancer is the leading cause of cancer-related death in Mexico, with most deaths being related to locally advanced or metastatic disease at diagnosis. Epithelial-mesenchymal transition (EMT) is one of the steps that are indispensable for metastasis. Different factors trigger EMT, like TGF- $\beta$, EGF and interleukin 6 (IL-6), among others. EMT is characterized by $\mathrm{E}$-cadherin expression loss and $\mathrm{N}$-cadherin and vimentin expression. In this study, we investigated the role of IL-6 on EMT induction. Methods: MBCDF and MBCD17 primary breast cancer cell cultures were used. E-cadherin expression was measured by Western Blot. Cells were stimulated with IL- 6 to induce EMT. STAT3 activation was measured using phospho-specific antibodies, and E-cadherin expression was measured as EMT marker. Results: MBCDF and MBCD17 primary breast cancer cell cultures stimulation with IL-6 induced STAT3-Tyr705 phosphorylation without its total levels being altered; in addition, IL-6 cell-stimulation was shown to induce EMT, as evidenced by E-cadherin loss. Conclusions: The results of the present work suggest that IL-6 induces EMT in primary breast cancer cell cultures through STAT3 phosphorylation. (creativecommons.org/licenses/by-nc-nd/4.0/).
\end{abstract}

*E-mail for correspondence: mibarra.innsz@gmail.com (M. de J. Ibarra-Sánchez) ${ }^{\#}$ These authors contribute equally to this work. 


\section{INTRODUCTION}

Globally, breast cancer accounts for $25 \%$ of all malignancies in women, which places it as the most common in this group. In Mexico, it is the leading cause of cancer-related death in women since $2006^{1,2}$. In 2012, an increase in breast cancer incidence was reported, from $2 \%$ in 1980 to $5 \%$ being reported in $2010^{2}$. In 2009, the Mexican epidemiological surveillance system published a nation-wide incidence of 15 cases per 100,000 population, with the highest incidences being noted in Distrito Federal (now Mexico City), Coahuila and Nuevo León, with 17, 18 and 14 cases per 100,000 population, respectively, while the state with the lowest documented incidence was Chiapas, with 1.5 cases per 100,000 population. With regard to these data, it should be noted that in Mexico there is no national cancer registry, and the reported figures are therefore only an estimate of breast cancer actual situation in the country.

Breast cancer molecular study has enabled to classify the disease in different subtypes, with the purpose to translate this information into targeted therapies and define prognostic groups. In the past few decades, this classification has undergone modifications that represent research advances and adaptations for global classification criteria, with a specific value of Ki67 being eliminated and clinical parameters and multi-parametric molecular markers being added, as main modifications. Hence, luminal tumors, characterized by hormone receptor expression and HER2 non-expression, which are subdivided in luminal A-type, such as those tumors where immunohistochemistry analysis reveals estrogen receptor (ER) and progesterone receptor (PR) high expression, a clearly low Ki67 determination, and tumors classified by size as T1 and T2 and involvement of 0 to 3 lymph nodes; if access to multi-parametric molecular markers determination is available (Oncotype DX ${ }^{\oplus}$, MammaPrint ${ }^{\oplus}$ ), the result should be a favorable risk assessment. This subtype accounts for $40 \%$ of breast cancer cases and is associated with favorable prognosis. Luminal B subtype is characterized by hormone receptor low expression, clearly high Ki67, nodular involvement higher than 3 lymph nodes, histological grade 3 (poorly differentiated tumor), extended lymph-vascular invasion and bulky tumors (T3); this type accounts for $20 \%$ of breast cancer cases and is associated with a mortality risk of $1.96(95 \%$ $\mathrm{Cl}$ : $1.08-3.54)$. HER2 overexpression ( $25 \%$ of tumors) or the lack of estrogen receptor (ER), progesterone receptor (PR) and HER2 expression is defined as "triple-negative" (15 to $20 \%$ of breast cancer cases), these two groups considered of poor prognosis, with a mortality risk of $7.39(95 \% \mathrm{Cl}$ : 1.72-31.77) and $12.41(95 \% \mathrm{Cl}: 5.82-26.49)$, respectively. It should be noted that, in women younger than 40 years, the factors that more negatively influence on overall survival are lymph node infiltration or triple-negative molecular subtype ${ }^{3,4}$.

The introduction of targeted therapies to specific molecules such as the epidermal growth factor receptor (Hercep$\operatorname{tin}^{\oplus}$, TDM-1, Pertuzumab ${ }^{\circledast}$ ), have achieved objective tumor responses of up to $70 \%$ when combined with chemotherapy, and have improved overall survival. This is only effective for a selected group of patients; however, the disease has been observed to be able to progress over time and to acquire the capability to generate metastasis to other sites ${ }^{5}$.
One of the first events in tumor cell dissemination is the loss of the epithelial phenotype through drastic changes in the cytoskeleton. These series of events are known as epithelial-mesenchymal transition (EMT), which is a process whereby epithelial cells undergo biochemical changes to transform into mesenchymal cells; intercellular bonds are lost and cells therefore become elongated and non-polarized, which allows them to move across the extracellular matrix, by means generating a new set of tumor cells with different polarity than the epithelium they originated in ${ }^{6,7}$. EMT is not a process exclusive to tumor cells, but it has been described in 3 different biological scenarios: Type 1 EMT participates in embryo implantation, embryogenesis and organogenesis; type 2 EMT is associated with wound heal ing, tissue regeneration and fibrosis in organs such as the kidney; and type 3 EMT is involved in tumorigenesis, which accounts for the progression of a carcinoma in situ to an invasive one ${ }^{8}$, and has also been associated with resistance to apoptosis and chemotherapeutic drugs ${ }^{9}$. The role of EMT in cancer is already well established, and it is assumed to be an indispensable component for metastasis ${ }^{10}$. Once tumor cells have reached the distant metastasis site, they return to the primary tumor phenotype through a process known as mesenchymal-epithelial transition (MET). Therefore, EMT steps appear to be reversible, thus indicating the existence of dynamic components in tumor progression ${ }^{11}$.

Several markers implied in EMT have been described, and one of the most widely studied is E-cadherin expression decrease, which is a protein involved in cell-cell adhesion that lowered its levels to the minimum with an exchange for $\mathrm{N}$-cadherin. There are other markers that are positively regulated in EMT, such as: vimentin, fibronectin, smooth muscle actin; transcription factors such as Snail, Twist, Slug and ZEB; in addition to growth factors such as platelet-derived growth factor (PDGF) ${ }^{12-15}$. Other cell-cell adhesion molecules such as claudin 3, 4, 7, $\alpha$-catenin, $\gamma$-catenin and occludins are negatively regulated in cells with the mesenchymat phenotype ${ }^{13,15}$. EMT is a dynamic process and is activated by different stimuli of the tumor microenvironment, which include growth factors, tumor cell-stroma interactions and hypoxia ${ }^{16}$. Signals that activate EMT include growth factors such as transforming-growth factor $\beta$ (TGF- $\beta$ ), hepatocyte growth factor (HGF), fibroblast growth factor (FGF), insulin-like growth factor 1 and 2 (IGF 1 and 2) and epidermal growth factor (EGF) ${ }^{17}$.

In the past few years, the influence of tumor microen vironment has started to be taken into account in tumor development and behavior. Cytokines and growth factors secreted by cells surrounding tumors have been observed to act as crucial agents in tumor cells biological processes such as proliferation, migration, invasiveness and metastasis. Owing to this, systemic and tumor microenvironment markers, which are related to innate and acquired immune response have shown to play a crucial role in anti-tumor response ${ }^{18}$. Tumor microenvironment is composed by different cell types, including tumor-associated fibroblasts, neuroendocrine cells, adipocytes, inflammatory, endothelial and lymphatic cells ${ }^{19}$. All these cells types contribute to tumor development by secreting growth factors, metalloproteinases and cytokines that are necessary for tumor development.

One of the cytokines that has been implied in tumor progression is interleukin-6 (IL-6). This is a pleiotropic cytokine 
that is produced by hematopoietic and epithelial cells. Since its identification in mononuclear cell cultures supernatant, its role in biological functions such as B cell differentiation and $T$ cell proliferation has been described ${ }^{22,23}$. In breast, kidney and prostate cancer, as well as in myeloma multiple, it has been correlated with poor prognostic and tumor aggressiveness. Recent studies characterize IL-6 as a VEGF positive regulator ${ }^{24,25}$. IL-6 circulating levels have been found to be 10 -fold higher in patients with breast cancer than in healthy women, with a correlation existing between higher levels of IL- 6 and breast cancer more advanced stag$\mathrm{es}^{24,26}$. The study of IL-6 in breast cancer cells in vivo has yielded controversial results: on one hand, its implication in doxorubicin resistance and in the promotion of the motility required for metastasis have been demonstrated ${ }^{27,28}$, and on the other, treatment with low-dose IL- 6 for 6 days has been shown to inhibit ER-expressing cells proliferation in vitro via apoptosis activation by DNA fragmentation ${ }^{29}$. IL-6 has also been implicated as an EMT promoter by inducing E-cadherin expression repression ${ }^{30}$.

IL-6 signaling occurs through interaction with its receptor (IL-6R), and membrane-binding glycoprotein gp130, which is bound to JAK1,2. JAKs are in charge to phosphorylate gp130 distal cytoplasmic domain, with this phosphorylation serving to recruit $\mathrm{SH} 2$ domain-containing proteins, such as STAT3, which is subsequently activated by tyrosine residue 705 (Tyr705) phosphorylation ${ }^{23,31-33}$, thus inducing its dimerization. STAT3 dimer translocates to the nucleus, which results in the transcription of its target genes. In IL-6-mediated EMT, STAT3 induces the expression of other transcription factors such as Snail, ZEB, Twist and Slug, which together generate $\mathrm{E}$-cadherin repression and $n$-cadherin and vimentin mesenchymal genes transcription, which generated cell bounds disintegration and epithelial polarity loss ${ }^{34}$. Recent reports highlight the oncogenic importance of persistent STAT3 activation, and propose IL- 6 autocrine production and paracrine stimulation as STAT3 constitutive activation mechanism. In tumor microenvironment, as well as in tumor invasive fronts, presence of inflammatory cells has been observed, which suggests IL-6 might be a tumorigenic agent through JAK/STAT pathway activation ${ }^{11,35}$. This places EMT as being indispensable for the generation of metastasis, with this process also being observed to be reversible, which allows the cell to return to an epithelial phenotype once the site of metastasis is reached ${ }^{10}$.

On the other hand, in our laboratory, EGF was found to induce EMT measured as E-cadherin decrease and vimentin expression through transcription factor Snail induction ${ }^{36}$. Given these backgrounds, in the present work we analyze IL-6 effect on EMT induction. A model of primary breast cancer cell cultures was used, where EMT was induced, and changes in epithelial markers (E-cadherin) were examined. The results showed that IL- 6 induces EMT with the characteristic E-cadherin loss.

\section{MATERIALS AND METHODS}

\section{Reagents}

Primary antibodies against E-cadherin, tubulin, pSTAT3 (Tyr705) and STAT3 were obtained from Santa Cruz Biotech- nology (Santa Cruz, CA), and E-cadherin was obtained from Cell Signaling Technology (Cambridge, MA). Anti-mouse or anti-rabbit secondary antibodies were acquired from Jackson ImmunoResearch (West Grove, PA). Interleukin-6 (IL-6) was obtained from PeproTech (RockyHill, NJ).

\section{Cell culture}

MBCDF and MBCD17 primary breast cancer cells, which were derived from a biopsy of the specimen resulting from a mastectomy performed in a patient with breast cancer (protocol approved by the Instituto Nacional de Ciencias Médicas y Nutrición Salvador Zubirán Ethics Committee, ref. 1549, BQ0-008-06/9-1). The cell cultures were maintained in RPMI-1640 medium supplemented with $10 \%$ bovine fetat serum, antibiotic and antimicotic (Invitrogen Corporation, Camarillo, CA) at $37{ }^{\circ} \mathrm{C}$ in a moisturized atmosphere with $5 \% \mathrm{CO}_{2}$.

\section{Cell stimulation}

IL-6-stimulation assays were carried out in order to assess STAT3 phosphorylation by Western blot, using a phospho-specific antibody against PSTAT3 Y705 in MBCDF and MBCD17 cells; for this, $1 \times 10^{6}$ cells were seeded in $60-\mathrm{mm}$ culture plates, maintained in RPMI-1640 medium supplemented with $0.1 \%$ bovine fetal serum. The cells were allowed to adhere overnight at $37{ }^{\circ} \mathrm{C}$ and $5 \% \mathrm{CO}_{2}$. Stimulation was applied with IL-6 $1 \mathrm{ng} / \mathrm{mL}$ for the following time intervals: $0,5,15$, 30 and 60 minutes.

For the EMT induction and reversion assays, MBCD17 epithelial cells, two experimental models were designed: for the first one, short times of EMT IL-6-mediated induction were used of $0,4,8,12$ and $24 \mathrm{~h}$.

\section{Immunoblot assay (Western Blot)}

Stimulated cells were lysated with a lysis buffer contain ing: HEPES $50 \mathrm{mM}$ (pH 7.4), EDTA $1 \mathrm{mM}, \mathrm{NaCl} 250 \mathrm{mM}, 1 \%$ Nonidet, NaF $10 \mathrm{mM}$ and $1 \mathrm{x}$ protease inhibitors (Complete, EDTA-free, Roche). $25 \mu \mathrm{g}$ total protein underwent denaturing polyacrylamide gel electrophoresis and were transferred to Immobilion-P PVDF membranes (Millipore Corp, Bedford, MA), which were blocked for 60 minutes in 5\% skim milk in $0.05 \%$ PBS-Tween. Then, they were incubated with the respective antibodies overnight at $4{ }^{\circ} \mathrm{C}$ with agitation. Subsequently, the membranes were incubated with the an ti-mouse or anti-rabbit-HRP antibodies, as appropriate, for 45 minutes. The signal was visualized by chemoluminescense using the Super Signal West Pico kit (Thermo, Rockford, IL) and was finally exposed to a Kodak radiographic film.

\section{RESULTS}

\section{IL-6 induces STAT3 phosphorylation in breast cancer cells}

It is well established that IL-6 signals through STAT3 ativation $^{31}$. To demonstrate that IL- 6 was able to induce STAT3 activation in the primary breast cancer cell cultures, we stimulated the MBCDF and MBCD17 cells with $5 \mathrm{ng} / \mathrm{mL}$ of IL-6 for 


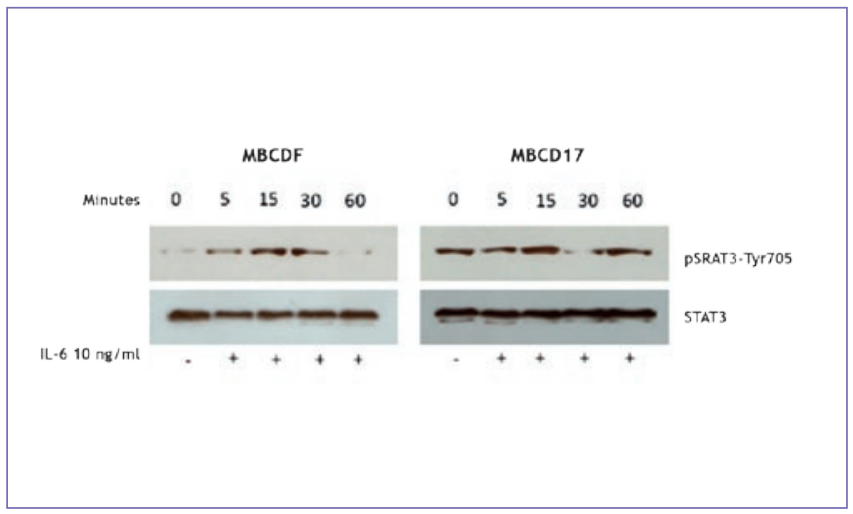

Figure 1. IL-6 induces STAT3 phosphorylation in MBCDF and MBCD17 breast cancer cells. Cells were treated with $5 \mathrm{ng} / \mathrm{mL}$ of IL-6 at $0,5,15$, 30 and 60 minutes; Western Blot was used to analyze STAT3 phosphorylation at Tyr705, with total STAT3 being used as control.

different time intervals. STAT3 activation was measured as STAT3 Tyr705 residue phosphorylation using phospho-specific antibodies. The results demonstrate that IL-6 induces STAT3 phosphorylation. Phosphorylation of STAT3 had an activation peak in MBCDF cells at between 15 and 30 min, whereas in MBCD17 cells, pSTAT3-Tyr705 activation peak occurred at 15 minutes (Fig. 1). These results confirm that, in these cultures, IL-6 signaling is mediated by STAT3 activation.

\section{IL-6 induces epithelial-mesenchymal transition}

Once we demonstrated that IL-6 induces STAT3 phosphorylation in primary breast cancer cell cultures, we investigated whether IL-6 stimulation elicits a decrease in E-cadherin expression as an EMT marker. IL-6 was found to induce a slight drop in $\mathrm{E}$-cadherin expression from $2 \mathrm{~h}$, which becomes more pronounced from $12 \mathrm{~h}$ and onwards. An anti-tubulin antibody was used as loading control. These results suggest that IL-6 induces rapid changes in E-cadherin expression, as a marker indicating that the tumor cell enters in EMT process (Fig. 2).

\section{DISCUSSION}

EMT is one of the most critical steps in the development of metastasis, and the description of the molecular mechanisms of which becomes activate and how it can be inhibited or reverted are therefore highly relevant to the development of new treatment strategies. In this work, we present evidence that IL-6 induces EMT through E-cadherin decrease in primary breast cancer cells. These data suggest that IL-6 is an important cytokine within the tumor microenvironment, which participates in breast cancer process of metastasis.

One of the main causes of mortality in breast cancer patients is metastasis. The process of metastasis includes several steps that a transformed cell has to complete to migrate to a distant site. Crucial steps of this process include EMT, which is characterized by cell polarity loss and invasive properties acquisition ${ }^{9,11}$. In order to be able to study metastatic cells properties, we developed an EMT in vitro

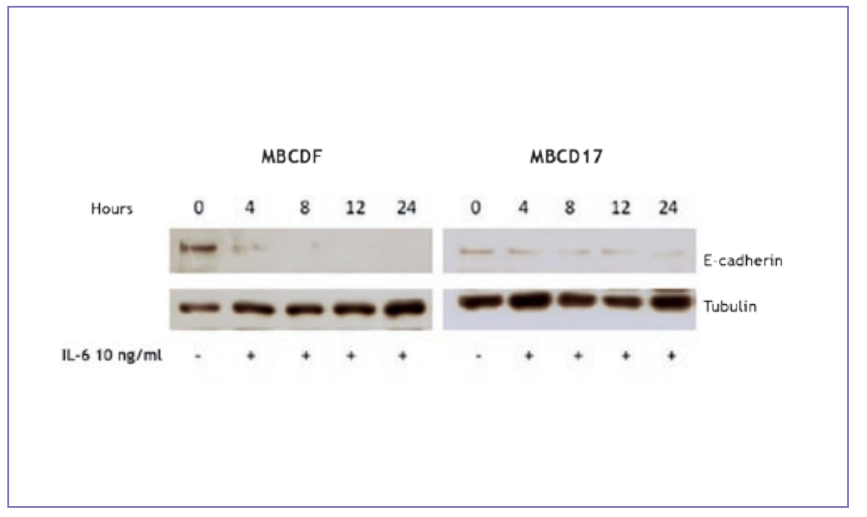

Figure 2. IL-6 induces E-cadherin expression loss in MBCDF and MBCD17 breast cancer cells. Cells were treated with $5 \mathrm{ng} / \mathrm{mL}$ of IL-6 at $0,4,8$, 12 and 24 hours; Western Blot was used to analyze E-cadherin expression (upper panel). An anti-tubulin antibody was used as loading control (lower panel).

model based on primary breast cancer cell cultures. EMT is a dynamical process, and it is activated by different stimulï of tumor microenvironment, including growth factors, tumor cell-stroma interactions and hypoxia ${ }^{16}$. EMT-activating signals include growth factors such as transforming growth factor beta (TGF- $\beta$ ), hepatocyte growth factor (HGF), fibroblast growth factor (FGF), insulin-like growth factors 1 and 2 (IGF 1 and 2) and epidermal growth factor (EGF) ${ }^{17}$. IL-6 is an inflammatory cytokine that has been associated with $\mathrm{EMT}^{30,37}$. In our primary breast cancer cell culture model, we stimulated epithelial marker-bearing cells (MBCDF and $M B C D 17)$ with IL-6 trying to induce EMT, demonstrating that IL-6 induces a decrease in E-cadherin expression in hours through STAT3 phosphorylation. These results demonstrate that, in our in vitro model of primary breast cancer cell culture, treatment with IL-6 is able to induce EMT, as demonstrated by the loss of E-cadherin expression.

\section{CONCLUSIONS}

In summary, our work demonstrates that IL-6 is a potent EMT inductor through STAT3 activation in primary breast cancer? cell cultures. The implications of this suggest that the presence of IL-6 in the tumor microenvironment confers high metastatic potential. These data support the development of new therapeutic strategies for breast cancer treatment; as IL-6 inhibition could be, as an attractive approach to intervene with EMT.

\section{CONFLICT OF INTERESTS}

The authors declare no conflicts of interests.

\section{REFERENCES}

1. Ferlay J, Soerjomataram I, Dikshit R, et al. Cancer incidence and mortality worldwide: sources, methods and major patterns in GLOBOCAN 2012. International journal of cancer. Journal international du cancer. 2015;136:E359-386. 
2. Forouzanfar MH, Foreman KJ, Delossantos AM, et al. Breast and cervical cancer in 187 countries between 1980 and 2010: a systematic analysis. Lancet. 2011;378:1461-1484.

3. Coates AS, Winer EP, Goldhirsch A, et al. Tailoring therapies--improving the management of early breast cancer: St Gallen International Expert Consensus on the Primary Therapy of Early Breast Cancer 2015. Annals of oncology : official journal of the European Society for Medical Oncology / ESMO. 2015;26:1533-1546.

4. Haque R, Ahmed SA, Inzhakova G, et al. Impact of breast cancer subtypes and treatment on survival: an analysis spanning two decades. Cancer epidemiology, biomarkers \& prevention : a publication of the American Association for Cancer Research, cosponsored by the American Society of Preventive Oncology. 2012;21:1848-1855.

5. Hortobagyi GN. Developments in chemotherapy of breast cancer. Cancer. 2000;88:3073-3079.

6. Nantajit D, Lin D, Li JJ. The network of epithelial-mesenchymal transition: potential new targets for tumor resistance. Journal of cancer research and clinical oncology. 2014.

7. Thiery JP, Sleeman JP. Complex networks orchestrate epithelial-mesenchymal transitions. Nature reviews. Molecular cell biology. 2006;7:131-142

8. Kalluri R, Weinberg RA. The basics of epithelial-mesenchymal transition. The Journal of clinical investigation. 2009;119:1420-1428.

9. Micalizzi DS, Farabaugh SM, Ford HL. Epithelial-mesenchymal transition in cancer: parallels between normal development and tumor progression. Journal of mammary gland biology and neoplasia. 2010;15:117-134.

10. Christiansen JJ, Rajasekaran AK. Reassessing epithelial to mesenchymal transition as a prerequisite for carcinoma invasion and metastasis. Cancer research. 2006;66:8319-8326.

11. Hugo H, Ackland ML, Blick T, et al. Epithelial--mesenchymal and mesenchymal--epithelial transitions in carcinoma progression. Journal of cellular physiology. 2007;213:374-383.

12. Sethi S, Macoska J, Chen W, Sarkar FH. Molecular signature of epithelial-mesenchymal transition (EMT) in human prostate cancer bone metastasis. American journal of translational research. 2010;3:90-99.

13. Gurzu S, Turdean S, Kovecsi A, Contac AO, Jung I. Epithelial-mesenchymal, mesenchymal-epithelial, and endothelial-mesenchymal transitions in malignant tumors: An update. World journal of clinical cases. 2015;3:393-404

14. Wu ZQ, Li XY, Hu CY, Ford M, Kleer CG, Weiss SJ. Canonical Wnt signaling regulates Slug activity and links epithelial-mesenchymal transition with epigenetic Breast Cancer 1, Early Onset (BRCA1) repression. Proceedings of the National Academy of Sciences of the United States of America. 2012;109:16654-16659.

15. Rito M, Schmitt F, Pinto AE, Andre S. Fibromatosis-like metaplastic carcinoma of the breast has a claudin-low immunohistochemical phenotype. Virchows Archiv : an international journal of pathology. 2014;465:185-191.

16. Wang S, Li S, Yang X, et al. Elevated expression of T-lymphoma invasion and metastasis inducing factor 1 in squamous-cell carcinoma of the head and neck and its clinical significance. European journal of cancer. 2014;50:379-387.

17. Foroni C, Broggini M, Generali D, Damia G. Epithelial-mesenchymal transition and breast cancer: role, molecular mechanisms and clinical impact. Cancer treatment reviews. 2012;38:689-697.
18. Gajewski TF, Schreiber H, Fu YX. Innate and adaptive immune cells in the tumor microenvironment. Nature immunology. 2013;14:1014-1022.

19. Hanahan D, Coussens LM. Accessories to the crime: functions of cells recruited to the tumor microenvironment. Cancer cell. 2012;21:309-322.

20. Kessenbrock K, Plaks V, Werb Z. Matrix metalloproteinases: regulators of the tumor microenvironment. Cell. 2010;141:52-67.

21. Whiteside TL. The tumor microenvironment and its role in promoting tumor growth. Oncogene. 2008;27:5904-5912.

22. Schaper F, Rose-John S. Interleukin-6: Biology, signaling and strategies of blockade. Cytokine Growth Factor Rev. 2015;26:475-487.

23. Wolf J, Rose-John S, Garbers C. Interleukin-6 and its receptors: a highly regulated and dynamic system. Cytokine. 2014;70:11-20.

24. Bachelot T, Ray-Coquard I, Menetrier-Caux C, Rastkha M, Duc A, Blay JY. Prognostic value of serum levels of interleukin 6 and of serum and plasma levels of vascular endothelial growth factor in hormone-refractory metastatic breast cancer patients. British journal of cancer. 2003;88:1721-1726.

25. Lippitz BE, Harris RA. Cytokine patterns in cancer patients: A review of the correlation between interleukin 6 and prognosis. Oncoimmunology. 2016;5:e1093722.

26. Kozlowski L, Zakrzewska I, Tokajuk P, Wojtukiewicz MZ. Concentration of interleukin-6 (IL-6), interleukin-8 (IL-8) and interleukin-10 (IL-10) in blood serum of breast cancer patients. Rocz Akad Med Bialymst. 2003;48:82-84

27. Conze D, Weiss L, Regen PS, et al. Autocrine production of interleukin 6 causes multidrug resistance in breast cancer cells. Cancer research 2001;61:8851-8858.

28. Arihiro K, Oda H, Kaneko M, Inai K. Cytokines facilitate chemotactic motility of breast carcinoma cells. Breast cancer. 2000;7:221-230.

29. Chiu JJ, Sgagias MK, Cowan KH. Interleukin 6 acts as a paracrine growth factor in human mammary carcinoma cell lines. Clinical cancer research : an official journal of the American Association for Cancer Research. 1996;2:215-221.

30. Asgeirsson KS, Olafsdottir K, Jonasson JG, Ogmundsdottir HM. The effects of IL-6 on cell adhesion and e-cadherin expression in breast cancer. Cytokine. 1998;10:720-728.

31. Heinrich PC, Behrmann I, Muller-Newen G, Schaper F, Graeve L. Interleukin-6-type cytokine signalling through the gp130/Jak/STAT pathway: The Biochemical journal. 1998;334 ( Pt 2):297-314.

32. Kamran MZ, Patil P, Gude RP. Role of STAT3 in cancer metastasis and translational advances. BioMed research international. 2013;2013:421821.

33. Xiong $\mathrm{H}$, Hong $\mathrm{J}$, Du $\mathrm{W}$, et al. Roles of STAT3 and ZEB1 proteins in E-cadherin down-regulation and human colorectal cancer epithelial-mesenchymal transition. The Journal of biological chemistry. 2012;287:5819-5832.

34. Lamouille S, Xu J, Derynck R. Molecular mechanisms of epithelial-mes enchymal transition. Nature reviews. Molecular cell biology. 2014; 15:178-196.

35. Bromberg JF, Wrzeszczynska MH, Devgan G, et al. Stat3 as an oncogene. Cell. 1999;98:295-303.

36. Moreno-Jaime B., Esparza-López J, Castro-Sánchez A, et al. El factor de crecimiento epidérmico induce transición epitelio-mesénquima en cultivos primarios de cáncer de mama. Gaceta Mexicana de Oncología: 2016;15:10-15.

37. Johnston PG, Rondinone CM, Voeller D, Allegra CJ. Identification of a protein factor secreted by 3T3-L1 preadipocytes inhibitory for the human MCF-7 breast cancer cell line. Cancer research. 1992;52:6860-6865. 